\title{
Endometrial VEGF induces placental sFLT1 and leads to pregnancy complications
}

\author{
Xiujun Fan, ${ }^{1}$ Anshita Rai, ${ }^{2}$ Neeraja Kambham, ${ }^{3}$ Joyce F. Sung, ${ }^{1}$ Nirbhai Singh, ${ }^{4}$ Matthew Petitt, ${ }^{1}$ Sabita Dhal, ${ }^{1}$ Rani Agrawal, ${ }^{5}$ \\ Richard E. Sutton, ${ }^{6}$ Maurice L. Druzin, ${ }^{1}$ Sanjiv S. Gambhir, ${ }^{7}$ Balamurali K. Ambati, ${ }^{4}$ James C. Cross, ${ }^{2}$ and Nihar R. Nayak ${ }^{1,8}$ \\ 'Department of Obstetrics and Gynecology, Stanford University School of Medicine, Stanford, California, USA. ${ }^{2}$ Department of Comparative Biology and Experimental Medicine and Department of Biochemistry \\ and Molecular Biology, University of Calgary, Calgary, Alberta, Canada. ${ }^{3}$ Department of Pathology, Stanford University School of Medicine, Stanford, California, USA. ${ }^{4}$ John A. Moran Eye Center, University \\ of Utah, Salt Lake City, Utah, USA. ${ }^{5}$ Department of Anesthesia, Stanford University School of Medicine, Stanford, California, USA. ${ }^{6}$ Section of Infectious Diseases, Department of Internal Medicine, \\ Yale University School of Medicine, New Haven, Connecticut, USA. 'Department of Radiology and Department of Bioengineering, Stanford University School of Medicine, Stanford, California, USA. \\ ${ }^{8}$ Perinatal Research Initiative, Department of Obstetrics and Gynecology, Wayne State University School of Medicine, Detroit, Michigan, USA.
}

\begin{abstract}
There is strong evidence that overproduction of soluble fms-like tyrosine kinase-1 (sFLT1) in the placenta is a major cause of vascular dysfunction in preeclampsia through sFLT1-dependent antagonism of VEGF. However, the cause of placental sFLT1 upregulation is not known. Here we demonstrated that in women with preeclampsia, sFLT1 is upregulated in placental trophoblasts, while VEGF is upregulated in adjacent maternal decidual cells. In response to VEGF, expression of sFIt1 mRNA, but not full-length Flt1 mRNA, increased in cultured murine trophoblast stem cells. We developed a method for transgene expression specifically in mouse endometrium and found that endometrial-specific VEGF overexpression induced placental sFLT1 production and elevated sFLT1 levels in maternal serum. This led to pregnancy losses, placental vascular defects, and preeclampsia-like symptoms, including hypertension, proteinuria, and glomerular endotheliosis in the mother. Knockdown of placental sFIt1 with a trophoblast-specific transgene caused placental vascular changes that were consistent with excess VECF activity. Moreover, sFIt1 knockdown in VEGF-overexpressing animals enhanced symptoms produced by VEGF overexpression alone. These findings indicate that sFLT1 plays an essential role in maintaining vascular integrity in the placenta by sequestering excess maternal VEGF and suggest that a local increase in VEGF can trigger placental overexpression of sFLT1, potentially contributing to the development of preeclampsia and other pregnancy complications.
\end{abstract}

\section{Introduction}

Preeclampsia is a serious pregnancy disorder, causing an estimated $14 \%$ of pregnancy-related maternal deaths and $15 \%$ of premature births worldwide. It generally presents as a syndrome of vascular dysfunction, which include maternal hypertension, proteinuria, and glomerular endotheliosis. Despite its longrecognized public health significance and intense investigation, its pathogenesis is still poorly understood, and definitive treatment is limited to delivery to prevent further maternal or fetal complications from disease progression $(1,2)$.

It has long been known that the symptoms of preeclampsia are dependent on the presence of the placenta, leading to the theory that preeclampsia is caused, in part, by placental factors that enter the maternal circulation (1-3). The strongest evidence argues for the involvement of soluble fms-like tyrosine kinase (sFLT1; the more common name for the soluble form of VEGFR1) in the development of maternal symptoms in most cases (2-4). Circulating levels of sFLT1 are increased in most cases of preeclampsia, and the levels increase about 5 weeks before the onset of maternal symptoms, consistent with a causal relationship (5). Systemic overexpression of sFLT1 in animal models produces preeclampsia-like symptoms (6,

Conflict of interest: The authors have declared that no conflict of interest exists Submitted: May 6, 2014; Accepted: August 21, 2014.

Reference information: J Clin Invest. 2014;124(11):4941-4952. doi:10.1172/JCI76864.
7), and extracorporeal removal of circulating sFLT1 in preeclamptic patients improves symptoms (8), largely confirming the role of sFLT1 in the maternal aspects of the disease. Although sFLT1 is an endogenous antagonist of both VEGF and placental growth factor (PLGF) (3), the maternal symptoms of preeclampsia most likely result from interference with VEGF signaling in the mother (2, 9, 10). Glomerulus-selective deletion of Vegfleads to glomerular endotheliosis and proteinuria in mice (9), for example, and patients treated with anti-VEGF chemotherapies show hypertension and proteinuria, clinical symptoms similar to those of preeclampsia $(2,10)$.

Despite great interest in sFLT1 as a cause of preeclampsia and the potential for treating preeclampsia through manipulation of sFLT1 and/or VEGF levels, the cause of sFLT1 upregulation is not fully understood. Increasing evidence suggests that VEGF itself may regulate sFLT1 production. VEGF, acting through FLK1 (also known as VEGFR2), can directly stimulate the production of sFLT1 by modulating the alternative splicing of FLT1 transcripts (11). VEGF has been shown to stimulate sFLT1 production in several cell types, including human placental explants (12). Although several other factors, including hypoxia-inducible factor-1, angiotensin II, and adenosine, have been shown to stimulate sFLT1 production (13-15), these factors are also known to either stimulate VEGF production or augment VEGF functions; thus, they may regulate sFLT1 production through the VEGF signaling pathway. Several reports have suggested that there may be increased 

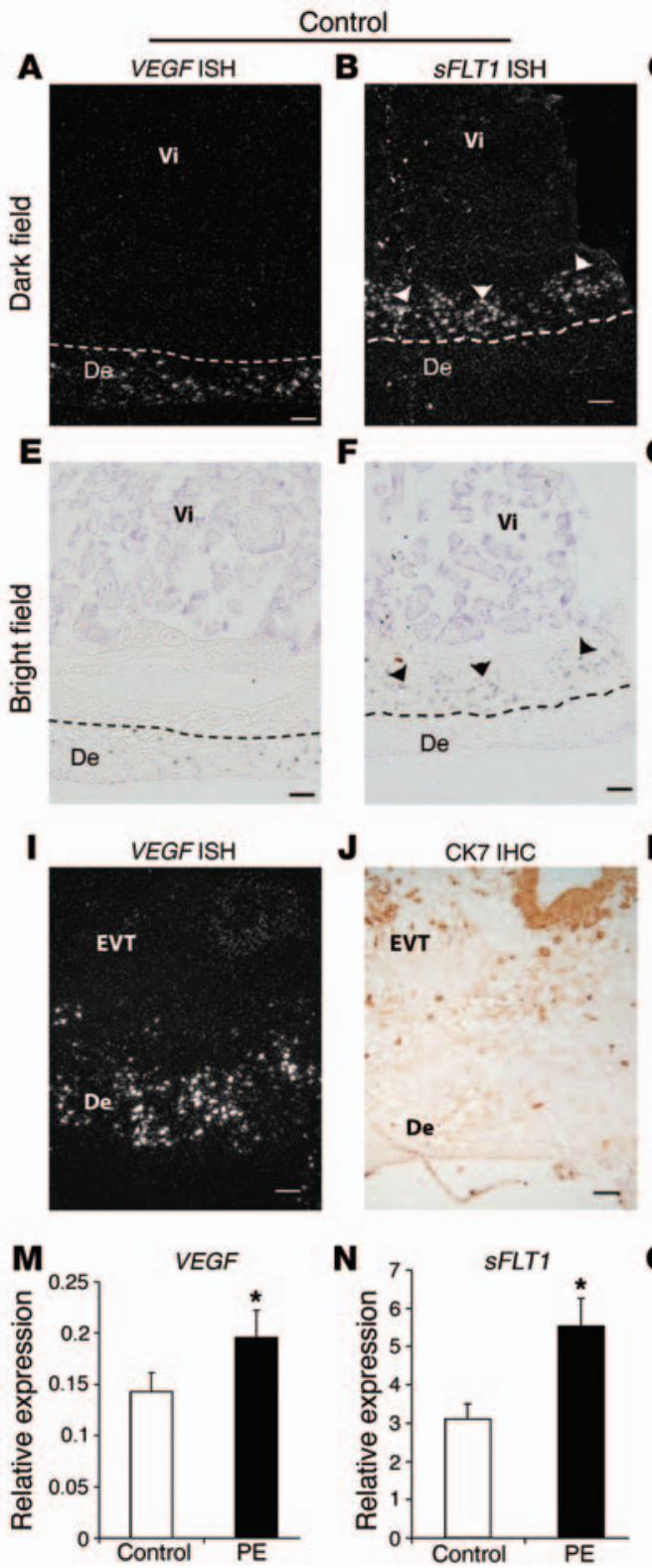

Vi

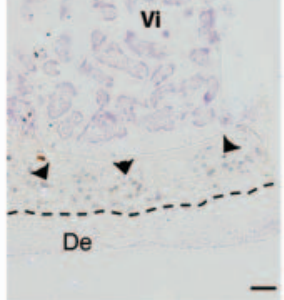

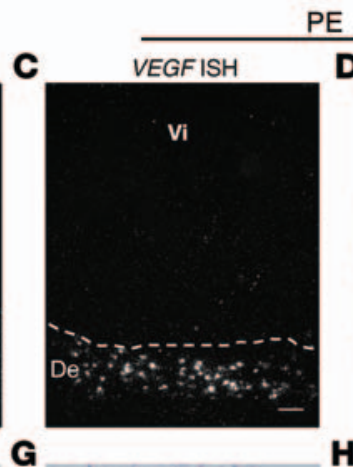
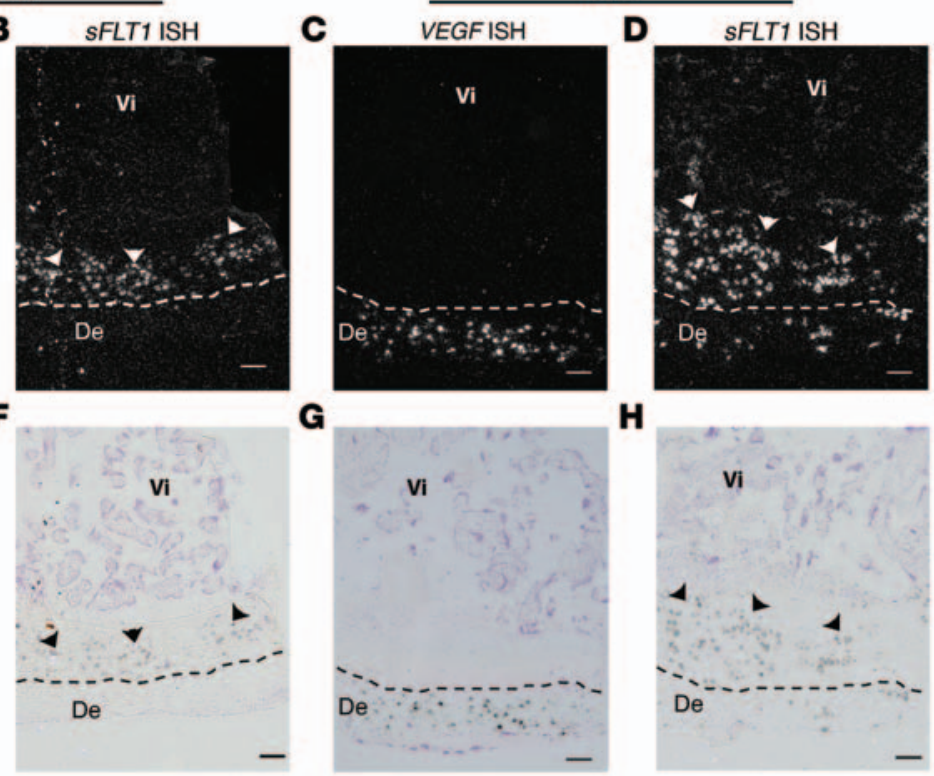

$\mathbf{K}$
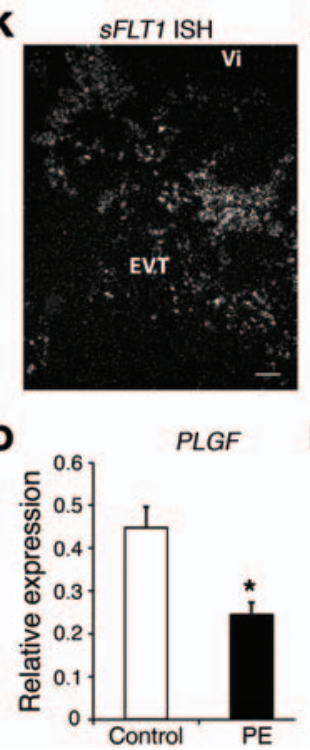

H

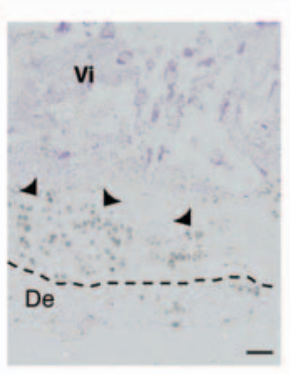

$\mathbf{L}$

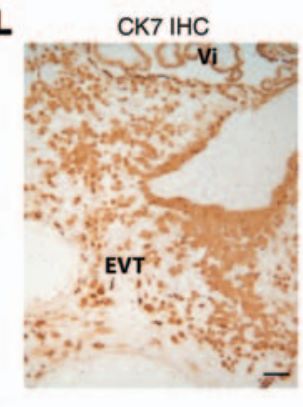

$\mathbf{P}$

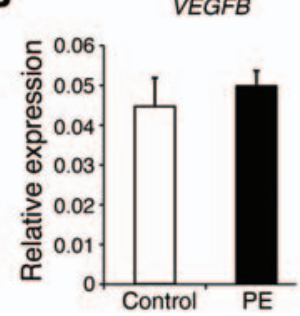

Figure 1. Increased VEGF expression in decidual cells and SFLT1 expression in adjacent EVTs in preeclamptic women. (A-H) VEGF and $S F L T 1$ mRNA expression by ISH (basal plate). Dashed lines demarcate the border between decidua (De) and placenta. Vi, villi. In specimens from both control and preeclamptic (PE) women, VEGF was expressed specifically in maternal decidual cells (A, $\mathbf{E}, \mathbf{C}$, and $\mathbf{G})$, and $S F L T 1$ in adjacent EVTs (arrowheads; B, F, D, and $\mathbf{H}$ ), but levels of both were dramatically elevated in preeclamptic specimens. (I-L) VEGF (I) and sFLT1 (K) ISH and CK7 IHC (J and L; sections adjacent to $\mathbf{I}$ and $\mathbf{K})$. (M-P) VEGF (M), sFLT1 (N), $\operatorname{PLGF}(\mathbf{O})$, and $\operatorname{VECFB}(\mathbf{P})$ total mRNA levels in placental basal plate samples from control and preeclamptic women, determined by qPCR. In preeclampsia, while levels of both VEGF and $S F L T 1$ significantly increased, PLGF significantly decreased, and VEGFB remained unchanged. Results are mean \pm SD. ${ }^{*} P<0.05(n=15)$. Scale bars: $100 \mu \mathrm{m}$. production of VEGF in preeclampsia $(12,16-20)$. While there are reports of both increased and decreased levels of serum VEGF in preeclampsia, these differences may be explained by differences in methods used for measurement $(6,18)$. Finally, exogenous VEGF administration in pregnant mice causes hypercoagulation in the placental circulation and hypertension, some of the clinical manifestations of preeclampsia (21).

Regardless of whether VEGF levels are altered in the serum of women with preeclampsia, local levels within the uterus and placenta are likely more relevant, since VEGF mostly becomes bound to plasma membrane and extracellular matrix after secretion and is widely viewed to function in a paracrine fashion (22-24). Therefore, we began our studies by determining the localization and relative expression levels of VEGF and sFLT1 at the decidualplacental interface in normal and preeclamptic pregnancies. We observed VEGF upregulation in decidual cells from preeclamptic pregnancies, along with a clear upregulation of sFLT1 in adjacent trophoblasts. This suggested that maternal VEGF-expressing cells in the decidua may influence sFLT1 expression in nearby placental cells. To test this, we used a new system to overexpress VEGF in the endometrium/decidua in mice and found that it caused increased sFLT1 production in the placenta and resulted in preeclampsia-like symptoms in the dam.

\section{Results}

Increase in VEGF and sFLT1 expression at the decidual-placental interface in preeclamptic women. We examined the cellular localization and regulation of both VEGF and sFLT1 at the decidual-placental interface (basal plate) using in situ hybridization (ISH) and quantitative real-time PCR (qPCR). Although the overall VEGF signal was low in the placental beds of control women, the signal in decidual cells was much higher than the signal in villous trophoblasts and extravillous trophoblasts (EVTs; Figure 1, A and E). Furthermore, in preeclamptic women, there was a strong increase in VEGF expres- 

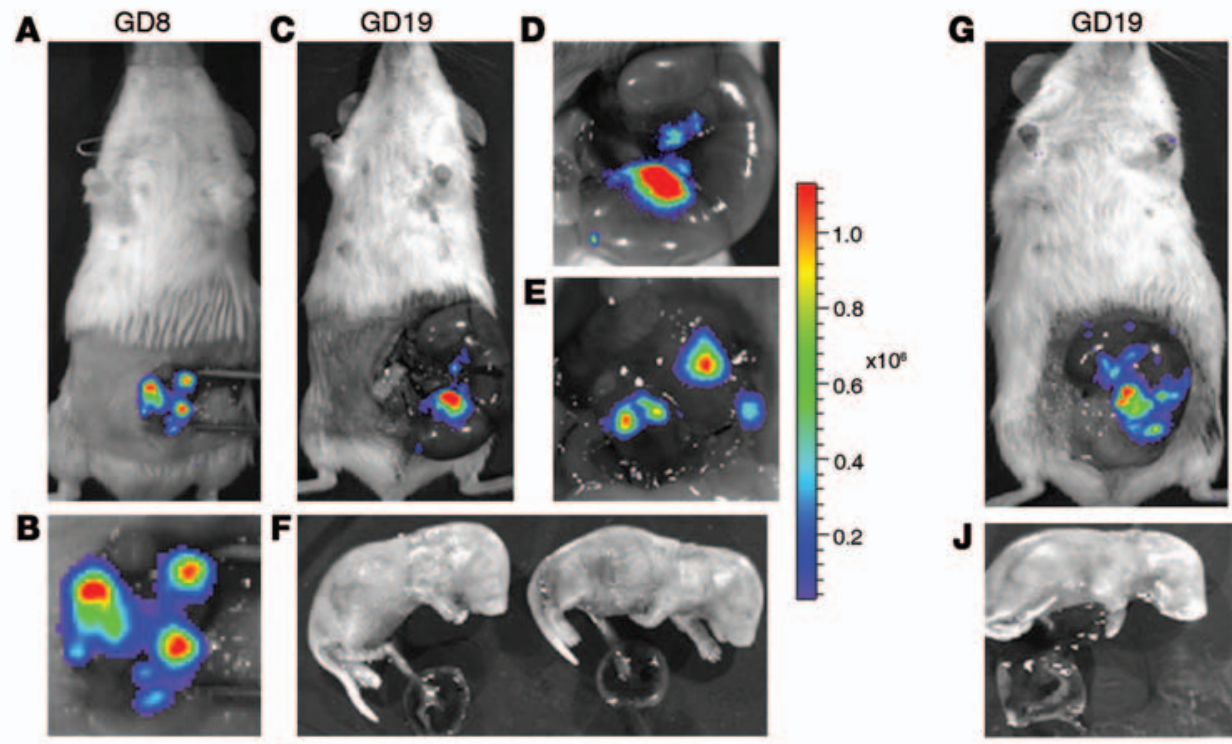

H

J

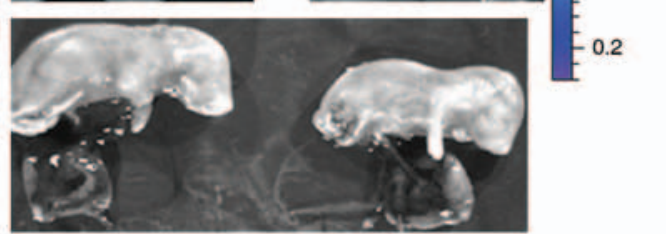

$\mathbf{K}$

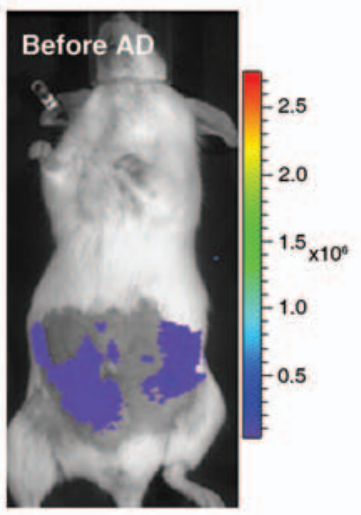

$\mathbf{L}$

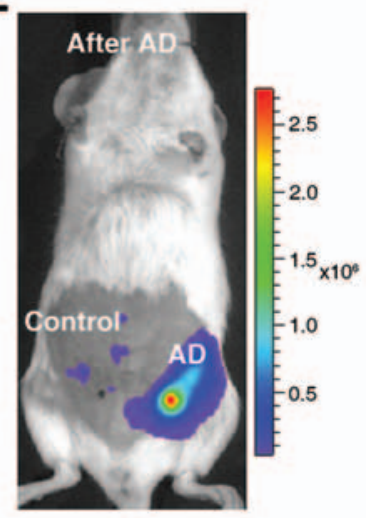

M
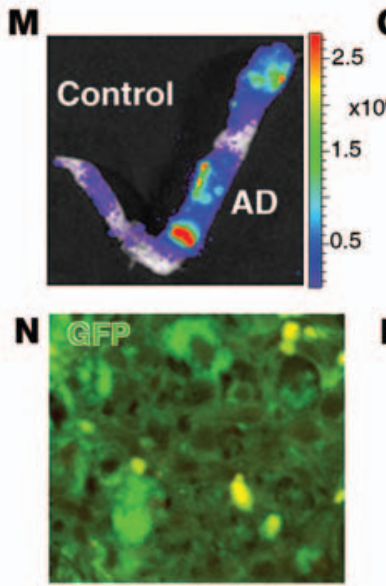

0

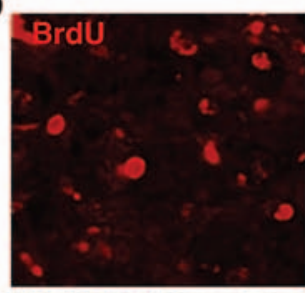

$\mathbf{P}$

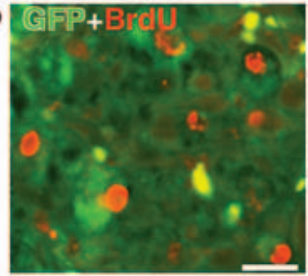

Figure 2. Development of an endometrium-specific gene delivery method: persistent transgene expression throughout pregnancy after LV-Fluc/GFP transfection of endometrial stromal cells. (A-F) First pregnancy. Bioluminescence imaging of uteri during laparotomy on GD8 (A and B) and GD19 (C-F). There was a marked increase in Fluc signal at the implantation sites on GD8 (A and B) that was maintained through GD19 (C and D). The signal persisted in the uterine wall $(\mathbf{E})$ after delivery of the fetuses and placentas $(\mathbf{F})$ by cesarean section. (G-J) Second pregnancy, following mating 30 days after first delivery. As in the first pregnancy, the signal increased during pregnancy (G-I) and persisted in the uterine wall (I) after removal of the fetuses and placentas (J) by cesarean section on GD19. (K-P) Artificial decidualization. (K) Live bioluminescence imaging before induction of artificial decidualization (AD) in LV-Fluc/GFP-transfected uterus (both horns). (L) Bioluminescence imaging 3 days after artificial decidualization induction in 1 horn. Note the dramatic increase in Fluc signal in the decidualized horn (M). (N-P) Colocalization (P) of GFP (N) and BrdU (0) to decidual cells; numerous GFP-expressing cells were BrdU positive, which suggests that the increase in Fluc signal during pregnancy may have resulted from proliferation of LV-Fluc/GFP-

transfected endometrial cells. Scale bar: $20 \mu \mathrm{m}(\mathbf{N}-\mathbf{P})$.

sion, primarily in decidual cells (Figure 1, C and G). Villous trophoblasts in some preeclampsia samples showed a moderate increase in signal, but this was always highly localized. In preeclamptic women, there was also a dramatic increase in $S F L T 1$ expression, primarily in the invading EVTs (Figure 1, D and H). Consistent with these results, our qPCR data from basal plate samples showed a 1.37-fold increase in VEGF and a 1.78-fold increase in sFLT1 mRNA levels in preeclamptic women relative to controls (Figure 1, M and N). Interestingly, in both control and preeclamptic women, VEGF was primarily expressed in maternal decidual cells and $s F L T 1$ in adjacent, invading EVTs (Figure 1, I-L), seemingly forming a barrier against maternal VEGF. These data suggest a potential regulatory interaction between decidual VEGF and placental sFLT1 production.
Endometrium-specific VEGF overexpression in mice. To explore the effects of increased endometrial VEGF expression, we used a mouse model of endometrial-specific VEGF overexpression, initially adapting previously described methods for transgene expression in the non-gravid mouse uterus using viral vectors (25, 26). However, our results indicated poor transfection efficiency and variable transgene expression (Supplemental Figure 1; supplemental material available online with this article; doi:10.1172/ JCI76864DS1). We next developed a method for consistent, highlevel expression of transgenes in the mouse endometrium during pregnancy that substantially overcame those limitations (referred to herein as Endo-VEGF mice; Figure 2). We found that the luminal epithelium was a barrier against virus delivery to underlying 
A
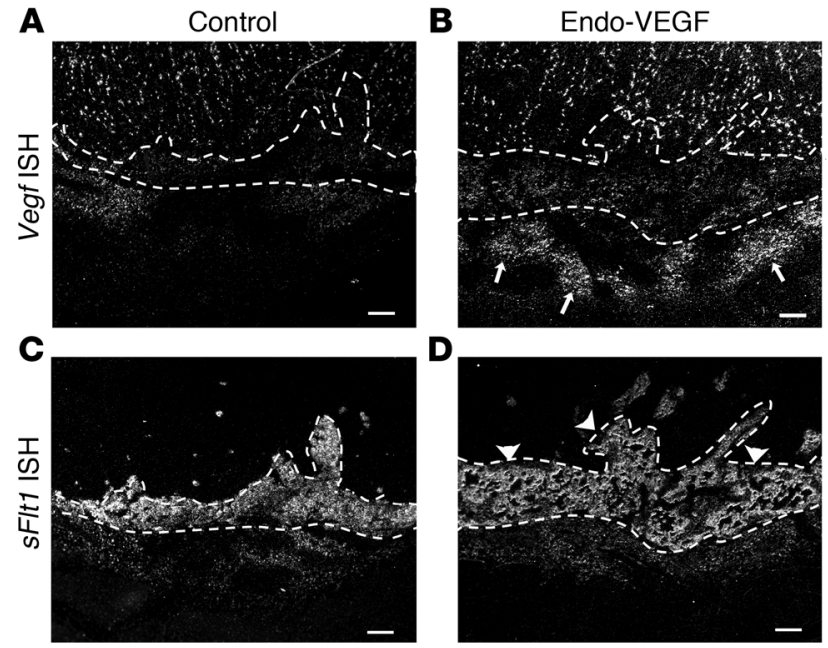

E

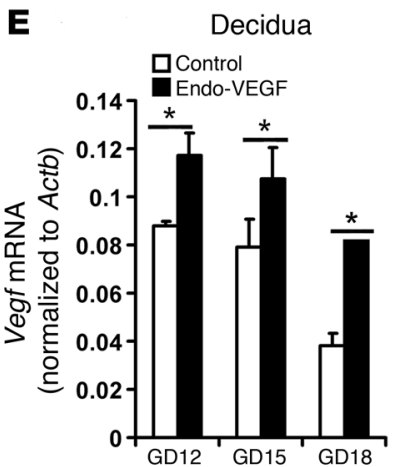

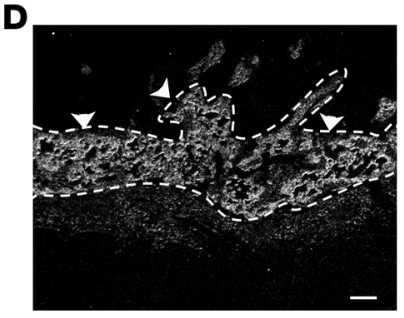

$\mathbf{F}$

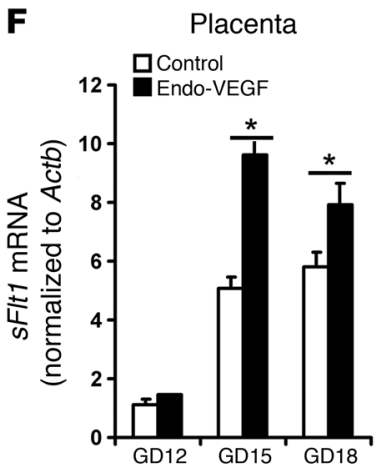

G

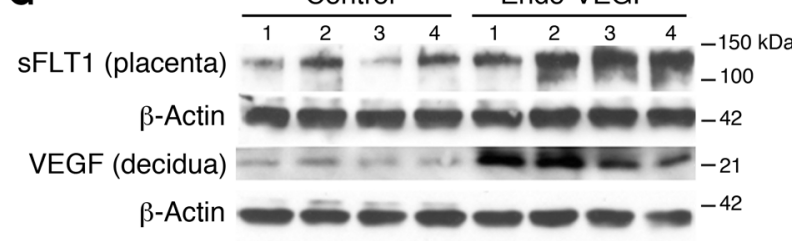

H

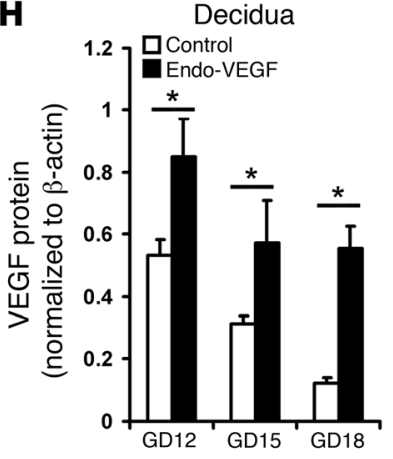

J

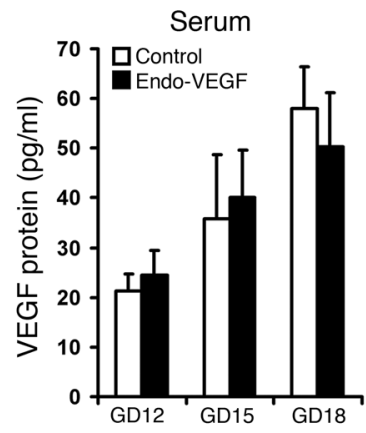

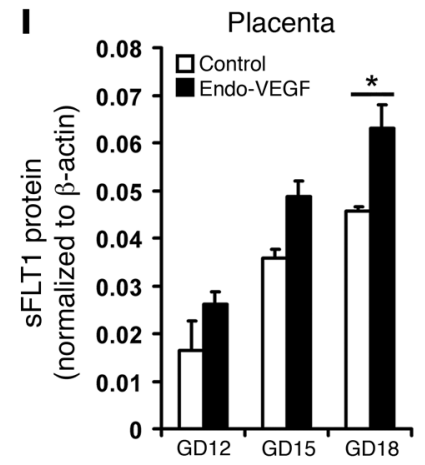

$\mathbf{K}$

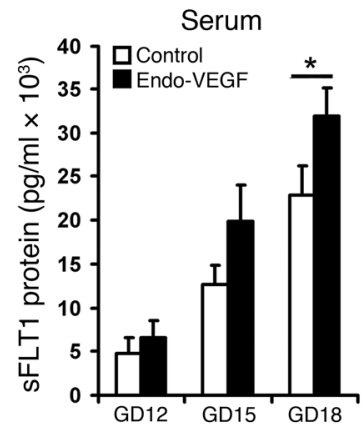

Figure 3. Endometrial VEGF overexpression leads to stimulation of placental sFLT1 production. (A-F) Vegf and sFlt1 mRNA expression by ISH (A-D) on CD18 (sections in $\mathbf{C}$ and $\mathbf{D}$ adjacent to sections in $\mathbf{A}$ and $\mathbf{B}$ ) and by qPCR on GD12, GD15, and GD18 in deciduas and placentas (E and $\mathbf{F}$ ). Increases in the Vegf signal (arrows) in deciduas (B and E) and in the sFlt1 signal (arrowheads) in placentas (D and F) were evident in Endo-VEGF animals. (G-K) Similarly, significant increases in decidual VEGF protein expression were observed by Western blot of Endo-VEGF tissues (G and $\mathbf{H}$ ). Corresponding increases in sFLT1 protein expression were found in the placenta (by Western blot; $\mathbf{G}$ and I) and in serum (by ELISA; K) of the same animals. No significant changes in circulating VEGF levels (bound plus free) were found by ELISA (J). Results are mean \pm SD. ${ }^{*} P<0.05$ ( $n=5$ ). Scale bars: $200 \mu \mathrm{m}(\mathbf{A}-\mathbf{D})$.

stromal cells, and that by removing it prior to transfection with lentiviral vectors, we could achieve consistent, high-level reporter gene expression specifically in underlying stromal (decidual) cells (Supplemental Figures 2 and 3). Luminal epithelium removal and subsequent viral infection did not affect the rate of conception or maintenance of pregnancy, and decidual reporter gene expression was maintained in Endo-VEGF mice through 2 successive pregnancies (Figure 2, A-J). Importantly, transgene expression was dramatically enhanced at implantation sites, likely from proliferation of the transfected endometrial cells during the process of decidualization (Figure 2, K-P), amplifying the transgene signal during pregnancy. We then used this method to evaluate the consequences of endometrial VEGF overexpression during pregnancy in mice (Figure 3). Examination of tissue samples by ISH, qPCR, and Western blotting revealed significant increases in VEGF protein and mRNA levels in decidual tissues from EndoVEGF animals throughout pregnancy (Figure 3, B, E, G, and H). Notably, although VEGF levels were increased locally, there was no increase in serum levels of VEGF (Figure 3J).
Endometrial VEGF overexpression adversely affects pregnancy outcome. Among 15 Endo-VEGF mice, all conceived normally, but the number of resorption sites was significantly increased and the number of viable fetuses decreased by the end of gestation (Figure 4, A and B). Most Endo-VEGF animals had transient vaginal bleeding between gestational day 10 (GD10) and GD15 (Figure 4H). Gross and histological examination of Endo-VEGF mouse specimens collected on GD12 and GD15 revealed that the anterograde vaginal bleeding likely resulted from hemorrhages primarily at the maternal-fetal interface at some of the implantation sites (Figure 4, I-P). Fetuses that survived to term had decreased weights, and the associated placentas were approximately 15\% lighter (Figure 4, C and D). Histological examination of the placentas revealed no significant changes in the morphologies and mean diameters of the trophoblast-lined spiral arteries (SpAs) at the junctional zone (JZ; Figure 5, A-D and M) or the endothelium-lined SpAs in deeper decidua (Figure 5, E-H). There was also no significant change in the depth of trophoblast invasion into the decidua or SpAs, as determined by cytokera- 

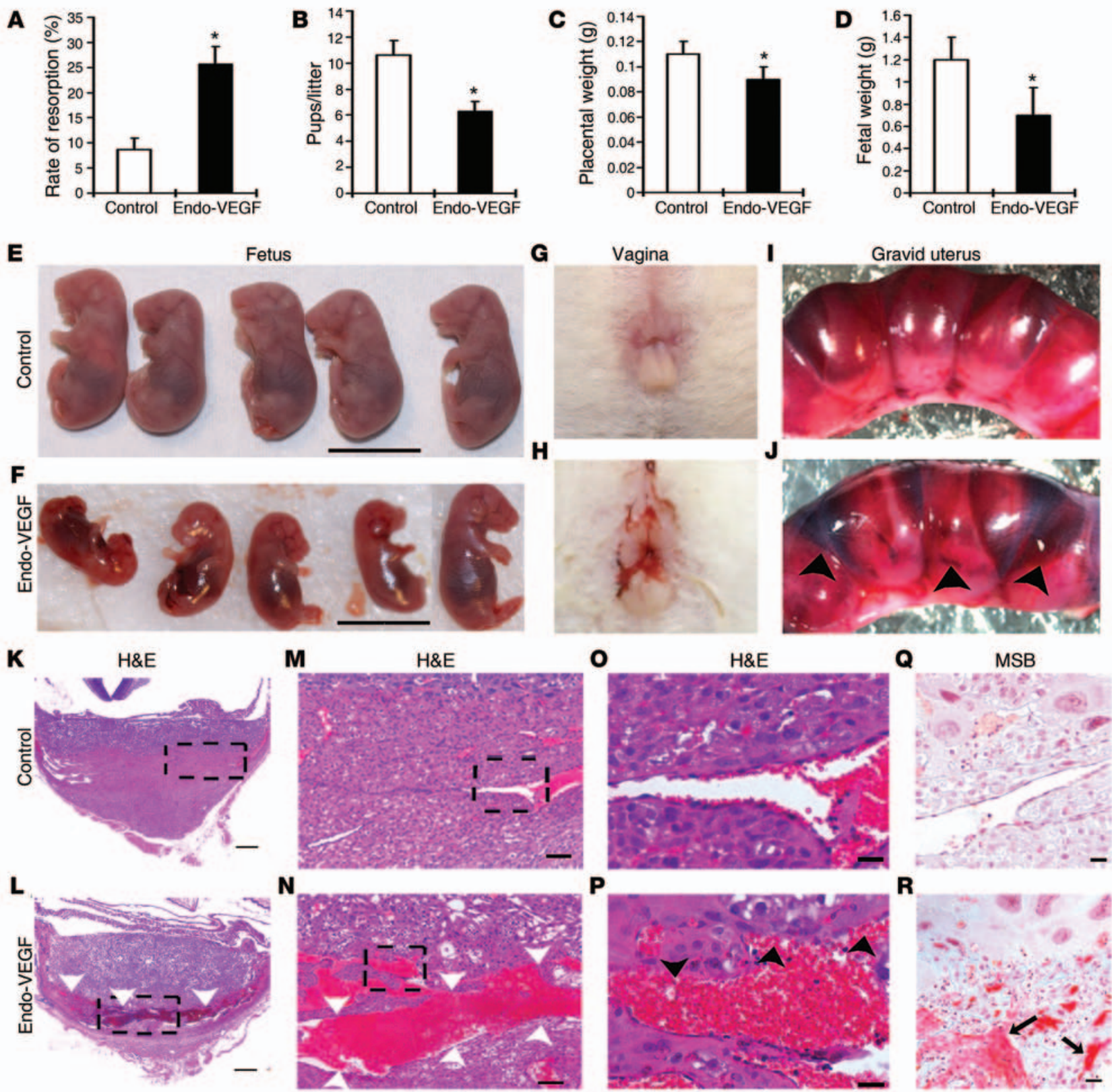

Figure 4. Overexpression of VEGF in the endometrium leads to increased pregnancy loss and widespread vascular deformity in the placenta, specifically in the venous channels and veins at the maternal-fetal interface. (A-F) VEGF overexpression significantly increased resorption rate (A) and significantly decreased number of pups per litter (B), placental weight (C), and fetal weight (D-F) on GD18. (G and $\mathbf{H}$ ) Vaginal bleeding occurred in most animals overexpressing VEGF, starting around CD10. (I and J) Gravid uteri in Endo-VEGF animals showed widespread hemorrhaging (arrowheads) compared with controls on GD12. (K-R) Histology of placental sections from control (K, M, O, and $\mathbf{Q})$ and Endo-VEGF animals (L, N, $\mathbf{P}$, and $\mathbf{R})$, assessed by H\&E (K-P) or martius scarlet blue (MSB) staining for extravasated fibrin ( $\mathbf{Q}$ and $\mathbf{R}$ ). Endo-VEGF animals showed severe dilation and congestion of venous sinuses and veins (white arrowheads) at the maternal-fetal junctions ( $\mathbf{L}$ and $\mathbf{N}$ ). Some of these venous sinuses exhibited focal, missing endothelial linings and hemorrhaging (black arrowheads) and extravasated fibrin (arrows) dissecting into the adjacent tissues (P and R). Results are mean \pm SD. ${ }^{*} P<0.05$ ( $\left.n=15\right)$. Scale bars: $10 \mathrm{~mm}$ (E and $\mathbf{F}) ; 500 \mu \mathrm{m}$ (K and $\mathbf{L}) ; 100 \mu \mathrm{m}$ (M and $\mathbf{N}) ; 20 \mu \mathrm{m}(\mathbf{O}-\mathbf{R})$.

tin 8 (CK8) immunohistochemistry (IHC; Figure 5, A-H and P). Within the placenta itself, the JZ was expanded (Figure 5O) due to changes in both trophoblast populations and vascular spaces. There was a significant increase in the number of glycogen trophoblasts (GlyTCs; about $83 \%$ of the expanded area), whereas there were no changes in the proportion of spongiotrophoblasts (Figure 5O). The central canals (which are downstream of the
SpAs and bring maternal blood into the placenta) and the maternal blood sinuses within the labyrinth zone appeared to be normal (Figure $5 \mathrm{M}$ ). The fetal vessels within the labyrinth that were close to the JZ were dilated and had significantly fewer vascular smooth muscle cells positive for NG2 and $\alpha$-smooth muscle actin ( SMA) (Supplemental Figure 4, A-O). In addition, the channels that bring maternal venous blood out of the labyrinth were dilated 


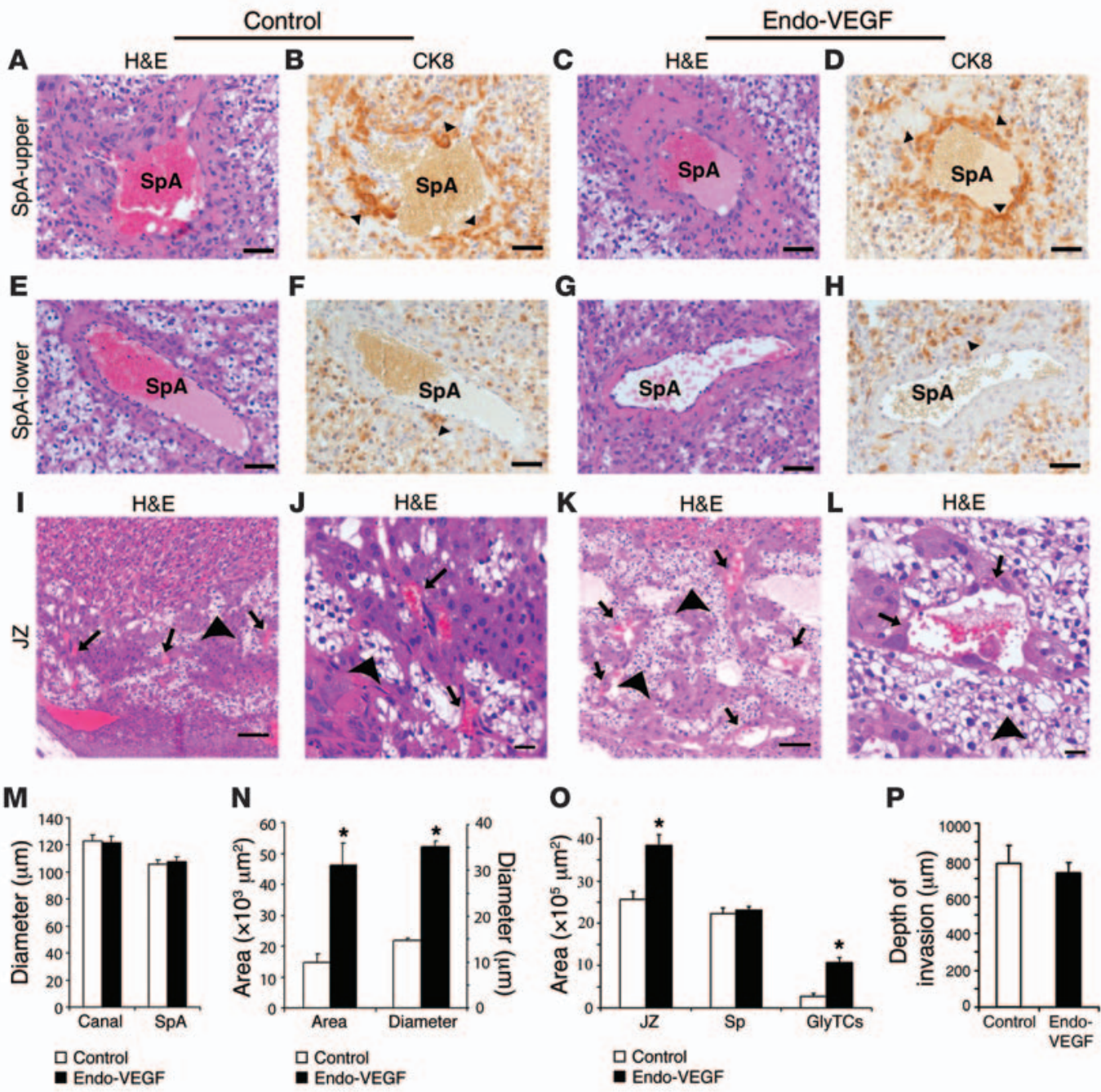

Figure 5. Effects of endometrial VEGF overexpression on placentas of surviving pregnancies that did not exhibit severe vascular changes. (A-H) Trophoblast-lined SpAs at the IZ (A-D) and endothelium-lined SpAs in deeper decidua, $300 \mu \mathrm{m}$ below the JZ (E-H). There were no marked changes between control and Endo-VEGF animals in either trophoblast-invaded (CK8-positive; arrowheads) SpAs ( $B$ and $\mathbf{D}$ ) or endothelium-lined SpAs (F and $\mathbf{H})$. (I-L) Expansion of the JZ, specifically GlyTCs (arrowheads), and dilation of venous channels (arrows) in Endo-VEGF animals ( $\mathbf{K}$ and $\mathbf{L}$ ) compared with controls (I and J). (M-P) Upon VEGF overexpression, there were no changes in $\mathrm{SpA}$ and central canal diameter (M), but significant increases were observed in cross-sectional area and diameter of venous channels (N) and in total JZ and GlyTC areas (0). (P) The depth of trophoblast invasion into the decidua was not significantly changed between groups. Sp, spongiotrophoblast. Results are mean \pm SD. ${ }^{*} P<0.05(n=15)$. Scale bars: $50 \mu \mathrm{m}(\mathbf{A}-\mathbf{H}) ; 100 \mu \mathrm{m}$ (I and $\mathbf{K}) ; 20 \mu \mathrm{m}$ ( and $\mathbf{L}$ ). and engorged (Figure 5, I-L and N), and the venous sinuses and veins at the maternal-fetal junction were severely dilated and congested (Figure 4, K-P). In places, VEGF overexpression was also associated with focal deposition of fibrin adjacent to some of these engorged vessels (Figure 4, Q and R).

Endometrial VEGF overexpression stimulates placental SFLT1 production and induces preeclampsia-like symptoms in later stages of pregnancy. Among the pregnancies surviving to GD18 in EndoVEGF mice, placental levels of full-length FLT1 and FLK1 protein and mRNA were not changed (Supplemental Figure 5). However, placental and serum sFLT1 were significantly increased in all Endo-VEGF animals (Figure 3, F, I, and K), which suggests that increased local VEGF can stimulate placental sFLT1 production. Because cells in the JZ, including GlyTCs, are the primary producers of sFLT1 in the placenta, the increase in SFLT1 expression may be due to expansion of these cells. The placenta appears to be the primary source of the increased serum sFLT1 levels, as circulating sFLT1 levels dropped to normal after placental delivery. To verify that VEGF acts directly on trophoblasts to influence sFLT1 expression, we cultured mouse trophoblast stem cells in differentiation medium in the presence of VEGF and found that it stimulated sFlt1 mRNA expression, but had no significant effect on expression of full-length Flt1 mRNA (Supplemental Figure 6).
In addition to increased sFLT1 levels, all Endo-VEGF animals with $>4$ live fetuses at term exhibited typical clinical signs of preeclampsia, including high blood pressure (starting on GD14) and proteinuria (Figure 6, A and B). Glomerular endotheliosis, a hallmark of preeclampsia, was also observed in several Endo-VEGF animals, as revealed by histological analysis and PAS staining (Figure 6, C-F). Although the lesions were more focal and segmental than in human preeclampsia, glomerular cellularity was significantly increased (control, $44.10 \pm 1.17$ cells/glomeruli; Endo-VEGF, $54.62 \pm 2.02$ cells/glomeruli; $P<0.05)$. Moreover, the symptoms of preeclampsia in our Endo-VEGF mice, including hypertension, completely disappeared after placental delivery (Figure 6A), similar to what is observed in preeclamptic women.

The detrimental effects of endometrial VEGF overexpression are enhanced by placental sFlt1 knockdown. To determine whether the adverse effects of endometrial VEGF overexpression were caused by VEGF itself or, instead, by induced placental sFLT1 production, we expressed an sFLT1-specific shRNA in the placenta using our previously reported lentivirus-based method for trophoblast-specific transgene expression (27). The same shRNA successfully knocked down sFlt1 in the cornea (28). A lentivirus expressing GFP alone was used as a control (Figure 7, A, C, and E). An sFLT1 shRNA-expressing lentivirus coexpressing GFP 

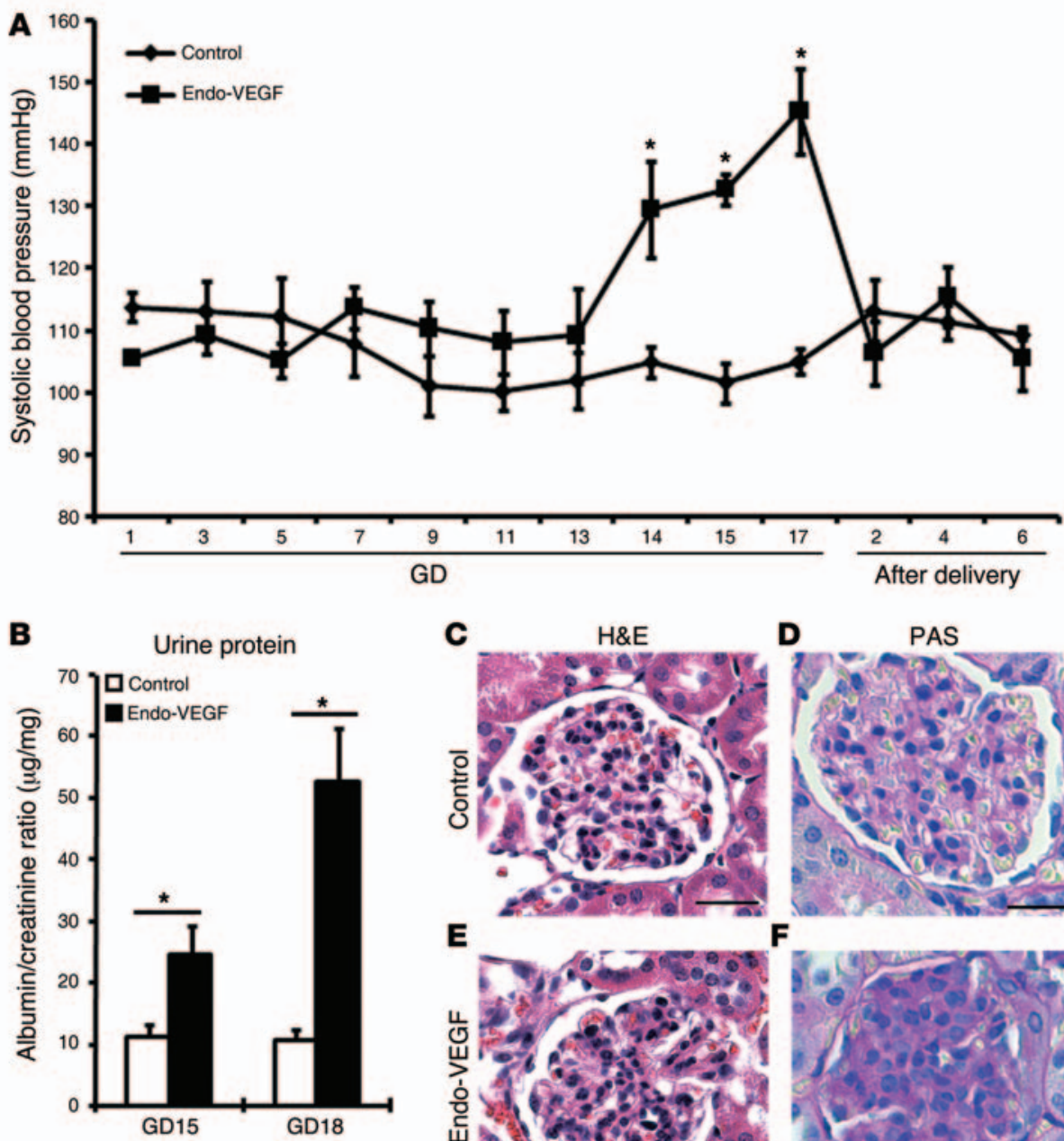

C

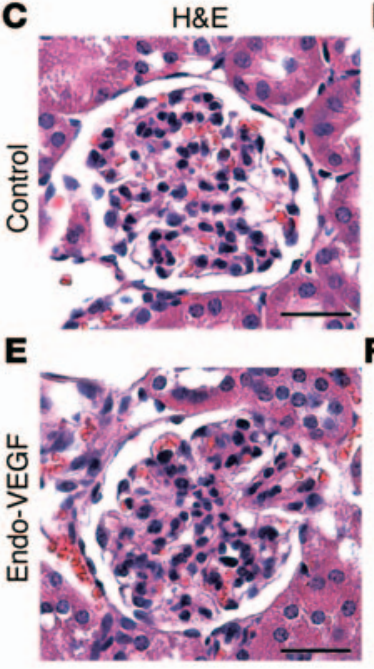

D

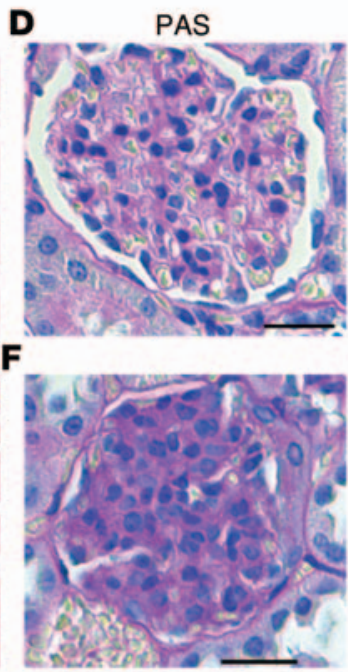

Figure 6. Endometrial VEGF overexpression leads to preeclampsia-like symptoms in mice. (A) Mean systolic blood pressure from control and Endo-VEGF animals with $>4$ live fetuses at term on different days of pregnancy. Endo-VEGF animals showed significant increases in blood pressure beginning at GD14, and blood pressure dropped to normal levels after delivery. (B) Significantly elevated albumin/creatinine ratio at both CD15 and GD18 in Endo-VEGF mice. (C-F) Staining of kidney sections with $\mathrm{H} \& \mathrm{E}$ (C and $\mathbf{E})$ and PAS (D and $\mathbf{F}$ ) revealed focal increase in glomerular cellularity and cytoplasmic swelling (endotheliosis) in Endo-VEGF animals. Results are mean \pm SD. ${ }^{*} P<0.05$ $(n=5)$. Scale bars: $20 \mu \mathrm{m}(\mathbf{C}-\mathbf{F})$.
(LV-sFLT1shRNA-copGFP) was used to transduce blastocysts and mark the cells expressing the sFLT1 shRNA (Figure 7, B, D, and F). After blastocyst transfer, pregnancy outcome, fetal and placental development, and sFLT1 levels were assessed. In mice expressing the sFLT1 shRNA, levels of placental sFLT1 protein and mRNA and circulating SFLT1 were greatly reduced, while levels of VEGF (total), PLGF, FLT1, and FLK1 were not affected (Figure 7, G-M, and Supplemental Figure 7). Although the sFLT1 shRNA expression in the trophoblast lineage of the placenta (Figure 7B) did not affect blastocyst implantation into the endometrium, after establishment of pregnancy, placental sFlt1 knockdown caused a significant increase in the number of resorptions (Figure 8, K and L). Most significantly, hemorrhages and enlargement of superficial vessels were evident by GD18 in about half of surviving fetuses with $s F l t 1$ knockdown and at placental-decidual junctions (Figure 8, A-D), similar to our findings in the Endo-VEGF model. Moreover, the maternal blood sinusoid spaces in the labyrinth layer were distended with sFLT1 shRNA expression (Figure 8, E-H). Placental sFlt1 knockdown-associated fibrin deposition in these spaces (Figure 8, E and F) and extravasated fibrin in adjacent areas (Figure 8, I and J) suggest that these dilated spaces were formed by cystic degeneration. These results suggested that, even in the absence of VEGF overexpression, sFLT1-producing cells in the JZ may inhibit local VEGF signaling in the placenta and act as a barrier to maternal VEGF entering the fetal circulation.

To explore the interaction of VEGF overexpression with $s F l t 1$ knockdown, we combined the treatments. Like the Endo-VEGF mice alone, animals with both VEGF overexpression and sFlt1 knockdown exhibited vaginal bleeding starting around GD10 and extensive hemorrhaging in placentas and deciduas (Figure 8, $\mathrm{M}-\mathrm{Q})$. Most fetuses were resorbed by GD15, with few surviving to GD16 before onset of excessive vaginal bleeding and termination of pregnancy or resorption. Placentas and deciduas collected on GD16 also showed vascular damage, similar to that in Endo-VEGF mice alone, but with widespread hemorrhage, severe engorgement of maternal venous sinuses and veins in the decidua and of maternal blood sinusoid spaces and fetal blood vessels in the labyrinth, and extensive fibrin extravasation (Figure 8, R-V). These results indicated that sFLT1 downregulation exacerbates the effect of endometrial VEGF overexpression on the local vasculature in the placenta. This suggested that the adverse effects of endometrial VEGF expression are caused by excess VEGF levels, not by the induced SFLT1, and that SFLT1 protects against excess locally produced VEGF at the maternal-fetal interface. 
A
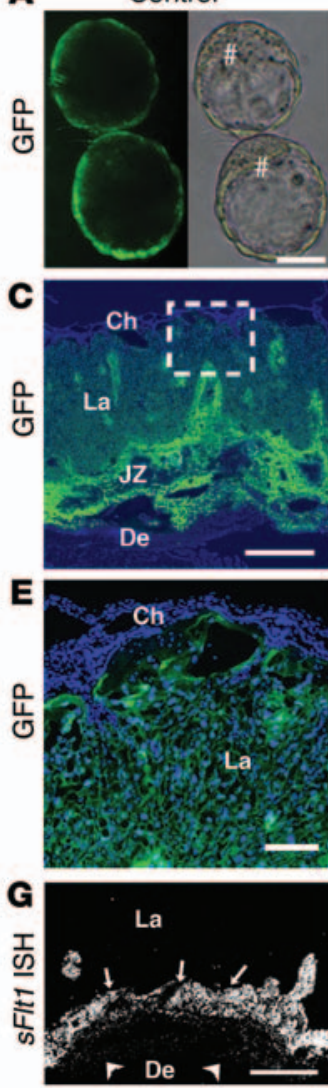

B
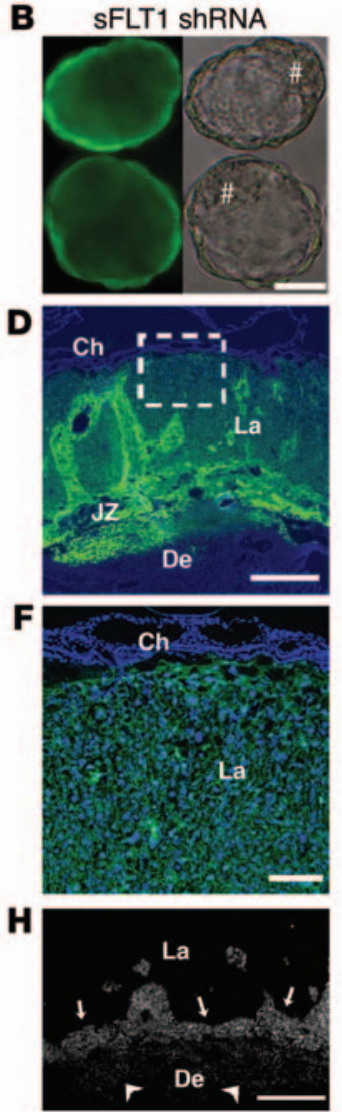

I

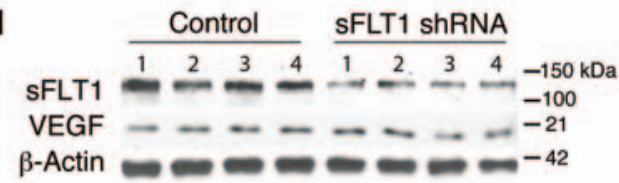

J

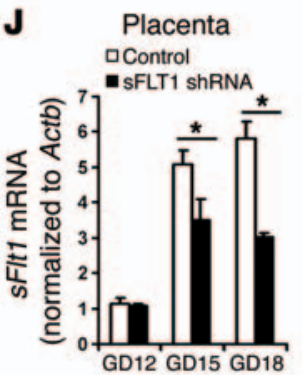

L Placenta

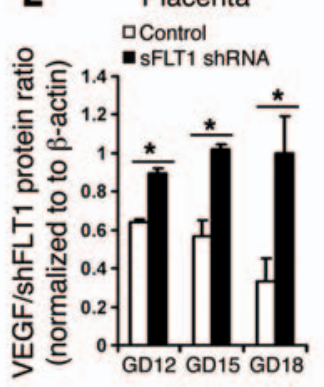

K

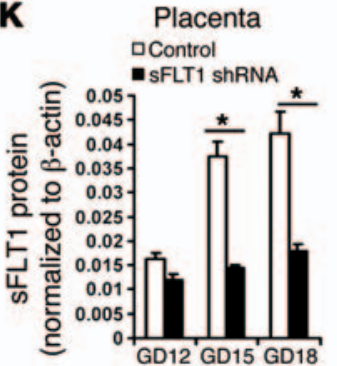

M

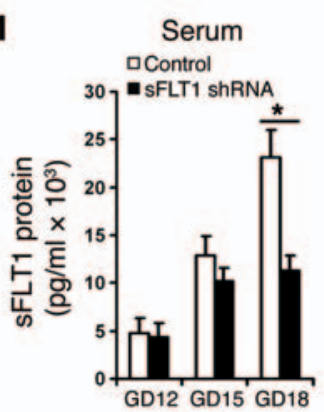

Figure 7. Effective sFIt1 knockdown in placentas by trophoblast-specific sFLT1 shRNA expression. (A and B) Blastocysts transduced with lentiviruses expressing GFP control (A) or both sFLT1 shRNA and GFP (B) expressed GFP (denoted by \#) in the trophectoderm, but not the inner cell mass. (C and D) Full-thickness placenta on GD18 showing GFP expression in trophoblast lineages from blastocysts transduced with control (C) and sFLT1 shRNA (D) viruses. (E and $\mathbf{F}$ ) Magnified images from $\mathbf{C}$ and $\mathbf{D}$ showing GFP expression in the labyrinth layer. ( $\mathbf{C}$ and $\mathbf{H}$ ) ISH showing dramatic reduction of $s$ Flt1 signal in sFLT1 shRNA-expressing placentas $(\mathbf{H})$ relative to controls $(\mathbf{C})$; signals were found primarily in GlyTCs and spongiotrophoblasts in the JZ (arrows) and the invading GlyTCs below the giant cell layer (arrowheads). Ch, chorionic plate; La, labyrinth layer. (I) Representative Western blots showing a substantial sFLT1 shRNA-mediated decrease in placental sFLT1 levels on GD18 without changes in VEGF levels. (J-M) qPCR of placental sFIt1 mRNA (J), quantification of placental sFLT1 protein by Western blot (K), and ELISA of maternal serum sFLT1 protein (M) revealed significant reductions at GD18 in placental sFIt1 knockdown animals, whereas (L) placental VEGF/sFLT1 protein ratio was significantly increased by sFlt1 knockdown. Results are mean \pm SD. ${ }^{*} P<0.05$ $(n=5)$. Scale bars: $20 \mu \mathrm{m}$ (A and B); $500 \mu \mathrm{m}$ (C, D, G, and $\mathbf{H}) ; 100 \mu \mathrm{m}$ (E and F).

\section{Discussion}

The results of the present study define an important regulatory relationship between increased local decidual VEGF production and increased sFLT1 expression in adjacent trophoblasts at the maternal-fetal interface in preeclampsia. Our evidence argues that, although the maternal symptoms of preeclampsia result from increased circulating levels of sFLT1, the upregulation of sFLT1 in placental cells may be a response to increased local levels of VEGF produced in maternal decidual cells. In addition, we found that sFLT1 plays a critical local role, even in normal pregnancies, by preventing damage to the placenta and fetus that would otherwise be caused by excess VEGF signaling. Our findings indicate that VEGF levels are tightly controlled at the maternal-fetal interface, at least in part through modulation by sFLT1. This result has potentially critical implications for the design of therapies for preeclampsia that manipulate sFLT1 and/or VEGF levels.

Our findings demonstrate that VEGF expression is increased in decidual tissue in preeclampsia. Increased expression and release of VEGF in placentas has been reported previously in women with preeclampsia $(12,16-19)$, and this correlates with the severity of the disease (17). Other studies have shown concomitant increase in serum (18) and kidney tissue (29) levels of VEGF and sFLT1 in preeclamptic women. However, studies on circulating levels of VEGF in preeclampsia have been inconsistent, with reports of both increased and decreased levels. It has been suggested that these inconsistencies are primarily due to differences in methods used for detection of free (unbound) vs. total (bound and unbound) circulating levels of VEGF $(6,18)$ : studies reporting decreased VEGF measured free VEGF (6), whereas studies reporting increased VEGF measured total VEGF $(16,18,19)$. In this respect, these studies support the hypothesis that while total VEGF production increases in preeclampsia, it is sequestered by the vast excess of sFLT1, thereby increasing levels of the bound form. Furthermore, our results, together with previous reports $(5,18)$, showed decreased production of PLGF and low abundance of VEGFB (the other ligands of sFLT1) in preeclampsia, which suggests that 
A
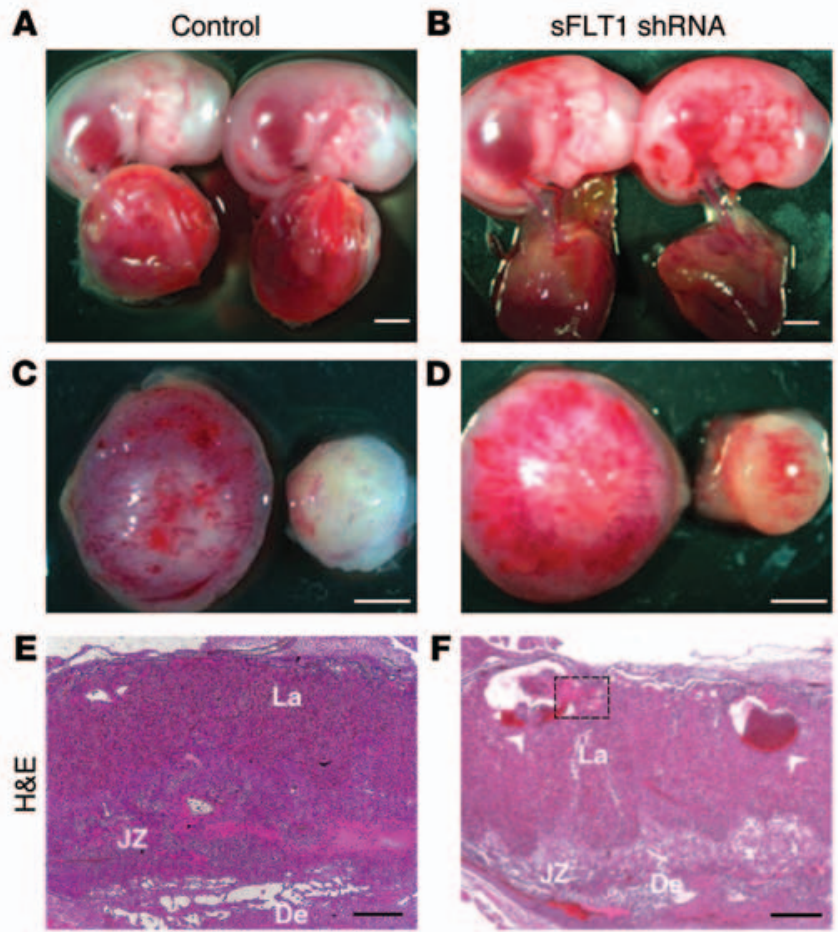

M

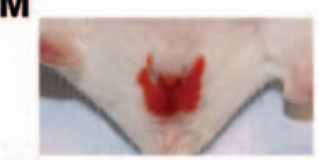

$\mathbf{N}$

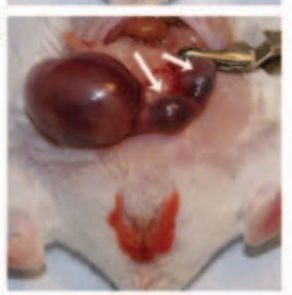

o

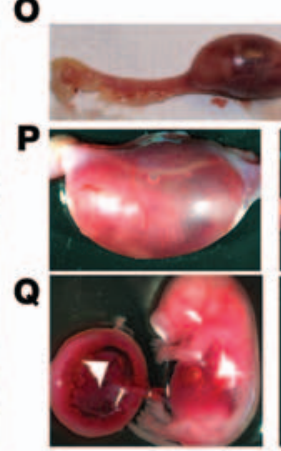

T

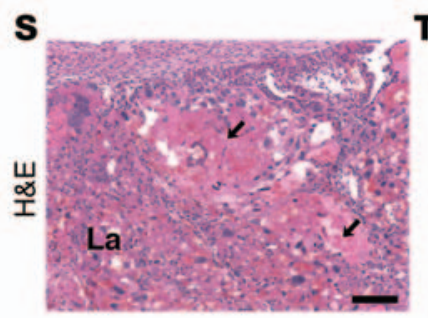

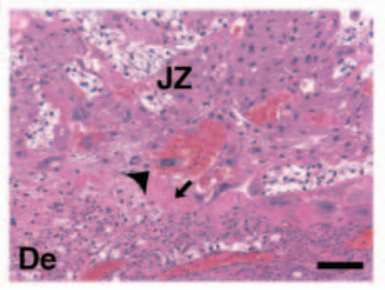

G

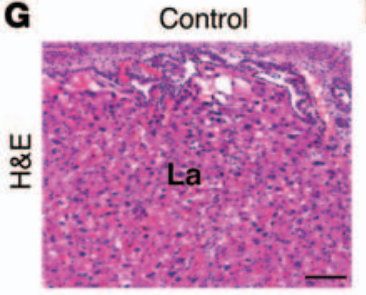

H
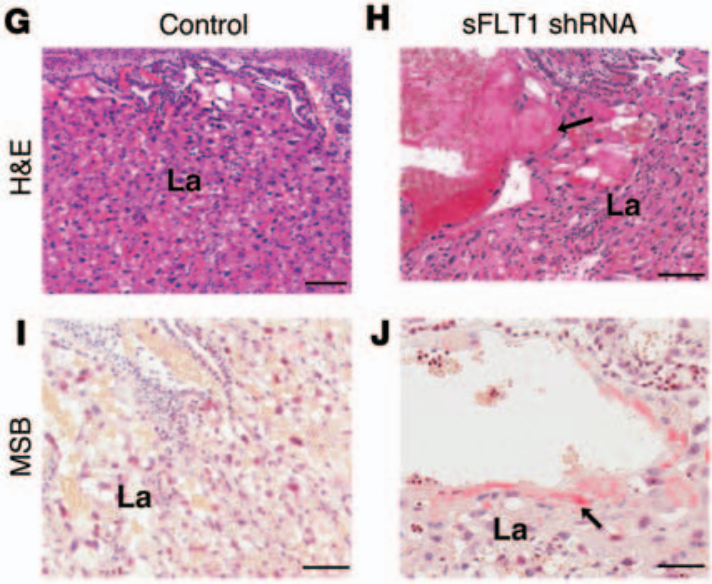

K

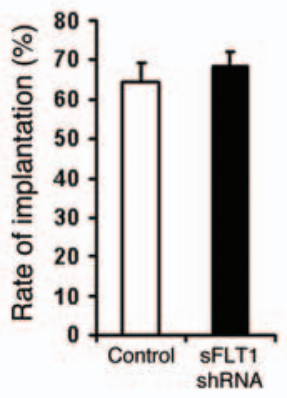

L

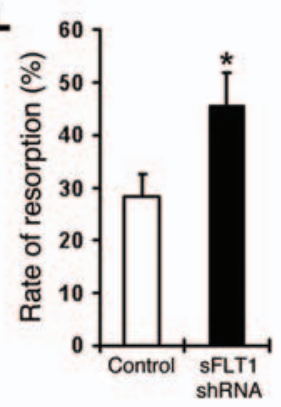

\section{$\mathbf{R}$}
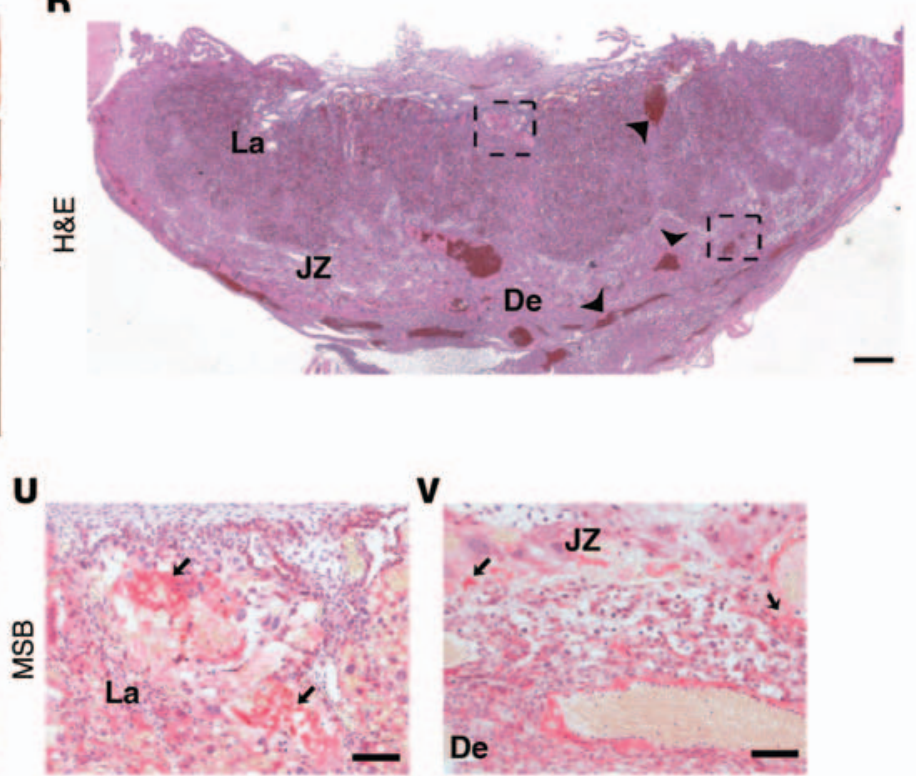

Figure 8. Effects of placental sFIt1 knockdown with or without endometrial VEGF overexpression. (A-L) Placental sFlt1 knockdown. Upon placentaspecific sFLT1 shRNA expression, widespread hemorrhaging in the fetus (B) and at the placental-decidual junction (D) was observed on GD18 compared with controls (A and $\mathbf{C}$ ). Histological examination of sFLT1 shRNA-expressing placentas revealed extraordinary dilation of some maternal blood sinuses (arrowheads) in the labyrinth (E and $\mathbf{F}$ ) and fibrin deposition (arrow) in these spaces ( $\mathbf{G}$ and $\mathbf{H}$ ). (I and J) MSB staining revealed extravasated fibrin (arrow) in adjacent areas. Placental sFlt1 knockdown did not affect implantation rate (K), whereas the fetal resorption rate significantly increased (L). (M-V) Placental sFIt1 knockdown enhanced the deleterious effects in Endo-VEGF animals. Pregnancies surviving to GD16 exhibited (M) excessive vaginal bleeding and ( $\mathbf{N}-\mathbf{P})$ termination of pregnancy or resorption (arrows denote resorption sites) as well as (Q) widespread and extensive hemorrhaging in fetuses and placentas (arrowheads) and in deciduas at the maternal-fetal junction (asterisk). Histological examination (R-T) revealed widespread dilation and congestion of maternal blood sinuses (arrowheads) in the labyrinth, venous sinuses, and veins at maternal-fetal junctions, and MSB staining (U and $\mathbf{V}$ ) demonstrated extensive fibrin extravasation (arrows) in the labyrinth and at the maternal-fetal junction. Results are mean \pm SD.

${ }^{*} P<0.05(n=15)$. Scale bars: $2 \mathrm{~mm}(\mathbf{A}-\mathbf{D}) ; 500 \mu \mathrm{m}$ (E, F, and $\left.\mathbf{R}\right) ; 50 \mu \mathrm{m}(\mathbf{G}-\mathbf{J}) ; 100 \mu \mathrm{m}(\mathbf{S}-\mathbf{V})$. 
among the 3 sFLT1 ligands, only VEGF increases in preeclampsia. Regardless of whether free VEGF levels are elevated in serum, we believe that it is the local levels of VEGF activity that matter in preeclampsia, as our mouse model showed that a moderate increase in decidual VEGF was sufficient to initiate sFLT1 release from the placenta, leading to the development of preeclampsia symptoms. Consistent with our observations, several studies that have carefully examined the relationship between VEGF level and biological outcome in vivo have found that VEGF has complex functions, that many cells are extraordinarily sensitive to VEGF, and that even modest changes in VEGF level can have dramatic consequences for development and physiology $(24,30)$.

Moreover, our results provided strong evidence that the increased circulating levels of sFLT1 in preeclampsia are primarily the consequence of local overproduction of VEGF at the decidualplacental interface. A recent report has indicated that in cultured endothelial cells, VEGF directly upregulates sFlt1 without influencing the expression of the membrane-associated full-length Flt1 (11). Furthermore, VEGF has been shown to stimulate release of sFLT1 from human placental explants in a dose-dependent manner (12), and VEGF treatment in pregnant mice can induce hypercoagulation and hypertension, some of the clinical manifestations of preeclampsia (21). Given that local VEGF level is a key determinant of VEGF function (22-24, 31), our findings that VEGF levels were increased specifically in the local microenvironment in the decidua and that sFLT1 was specifically increased in nearby trophoblasts in preeclamptic women strongly suggests a role for local VEGF in stimulating sFLT1 production in these cells. Although it is also possible that placental sFLT1 stimulates decidual VEGF expression in preeclampsia, we are not aware of any evidence in the literature to support this supposition.

Because endometrial VEGF overexpression led to increased placental sFLT1 levels, we investigated whether sFLT1 contributes to the placental changes in Endo-VEGF animals. The effects of placental sFlt1 knockdown in Endo-VEGF animals were similar to those of VEGF overexpression alone, but more pronounced and widespread, which suggests that the increased sFLT1 production in Endo-VEGF animals does not contribute to these effects, but rather that they result from the unopposed effects of excess VEGF activity. Furthermore, sFlt1 knockdown alone produced symptoms consistent with excess local activity of VEGF in other tissues (24, $28,31,32$ ) and with some of our findings in Endo-VEGF animals, which suggests that placental sFLT1 may play a critical role in neutralization of excess local VEGF at the maternal-fetal interface. We also found that placental knockdown of sFlt1 did not affect either blastocyst implantation or early placental development, similar to the previously reported finding that trophoblast-derived FLT1 is dispensable for establishment of the maternal-fetal interface (i.e., the early stages of pregnancy) (33). However, that study did not extend past midgestation, and we found here that placental knockdown of $s F l t 1$ caused widespread vascular abnormalities in both fetus and placenta and increased rates of fetal demise at later stages of pregnancy. Thus, our findings revealed a previously unknown requirement for sFLT1 for vascular homeostasis in the placenta and fetus in later stages of pregnancy.

Our present results and previously published reports (34-37) demonstrated similar patterns of expression and regulation of
VEGF and sFLT1 at the maternal-fetal interface in humans (basal plate and EVTs) and mice (JZ, spongiotrophoblasts, and GlyTCs) and showed that cytotrophoblasts were the main producers of sFLT1 at later stages of pregnancy in both species. The spatial expression and regulation of VEGF and sFLT1 in preeclamptic women also appeared to be identical to those of our Endo-VEGF murine model (i.e., dramatically increased VEGF expression in the decidual layer and sFLT1 in the adjacent EVTs; Figure 1). We also observed an expansion of the JZ (as reflected by glycogen cell number) in Endo-VEGF mice. A prior study in humans shows parallel expansion of the analogous EVT layer at the basal plate in preeclampsia (38), which could contribute to the overall increase in sFLT1 production. Although preeclampsia is often associated with shallow trophoblast invasion and consequent failure of SpA remodeling (1-3), we did not find changes in glycogen cell invasion of the decidua or remodeling and endovascular trophoblast invasion of SpAs in Endo-VEGF mice. However, this may not be an indication of a critical difference between humans and mice in terms of VEGF-sFLT1 regulation at the maternal-fetal interface. There is mounting evidence that failure of SpA remodeling can result from defects in decidualization and that it is not limited to preeclampsia; it is seen in several other pregnancy complications, such as intrauterine growth restriction and recurrent spontaneous abortion, suggesting it is an indicator of defects in multiple functional pathways (39). Therefore, it is possible that failure of SpA remodeling and increased placental sFLT1 production in preeclampsia may be independent processes; however, further work in primates is needed to examine whether these processes have a common origin in defects in placentation/decidualization and/or increased decidual VEGF levels.

Our data in mice also showed an association between VEGFinduced sFLT1 production and hypertension onset, as well as reversal of symptoms after delivery, similar to what is seen in preeclamptic women. Although numerous studies have implicated sFLT1 in the pathogenesis of hypertension in preeclampsia, the underlying molecular mechanism is not fully understood. Consistent with previous studies in both mouse models and preeclamptic women, the onset of hypertension in the Endo-VEGF animals in our study occurred well before term, when sFLT1 levels, although elevated, are similar to or even lower than the control levels at term $(40,41)$, yet control animals do not develop hypertension. It is likely that the sensitivity of hypertension to sFLT1 levels varies throughout pregnancy (30) and that the threshold level for development of hypertension at early stages is lower than it is at later stages.

Additionally, increases in VEGF and/or sFLT1 levels have been reported in other pregnancy diseases, such as intrauterine growth restriction, recurrent early pregnancy loss, and abruptio placentae (42-44). Some of the clinical symptoms associated with these disorders fit well with the symptoms manifested by our Endo-VEGF mice at different stages of pregnancy - for example, hemorrhages and early pregnancy loss associated with higher levels of VEGF and lower fetal weight and development of preeclampsia-like symptoms in pregnancies that continue to term. However, the molecular mechanisms underlying these pregnancy disorders are poorly understood, and future studies should examine the involvement of VEGF-sFLT1 signaling in the development of pathology in these pregnancy diseases. 
In summary, although VEGF is essential for normal embryonic development, we have shown that mild elevation of local VEGF levels during early pregnancy can cause severe placental vascular damage and embryonic lethality. Our results also showed that modest local increases in VEGF could also be a primary trigger for elevation of placental sFLT1 expression, leading to the hallmark symptoms of preeclampsia. We believe that placental sFLT1 plays an essential role in placental functions and that overexpression of sFLT1 in preeclampsia, although damaging to the mother, serves a critical protective function for the placenta and fetus through its sequestration of maternal VEGF. Considering current efforts to treat preeclampsia through systemic administration of VEGF or extracorporeal removal of circulating sFLT1, our results suggest that mild deviations from normal VEGF and sFLT1 levels during pregnancy could have serious consequences and underscore the need for caution when manipulating their levels for therapeutic purposes.

\section{Methods}

Human subjects. In total, 25 preeclamptic subjects and 34 gestational age-matched normal subjects (without preeclampsia or any other pregnancy complication) were recruited. For details of subject selection and clinical diagnosis of preeclampsia, see Supplemental Methods.

Animals. CD-1 (Charles River) female mice (8-12 weeks of age) were mated with fertile or vasectomized males (10-16 weeks of age) to induce pregnancy or pseudopregnancy, respectively; the day of vaginal plug detection was considered GD1 $(27,45)$.

Viral vectors. Lentivirus construction and production were described previously $(27,46,47)$. Briefly, LV-Fluc/GFP was prepared by incorporating the coding sequences for firefly luciferase (Fluc) and EGFP linked by an encephalomyocarditis virus internal ribosomal entry site (IRES) sequence $(27,46)$. The lentivirus encoding VEGF and EGFP (LV-VEGF/GFP) was constructed using a self-inactivating HIV-based vector (46), For preparation of the lentivirus encoding the shRNA targeting sFLT1 (LV-sFLT1shRNA), a sequence in the unique carboxyterminal region of $s F l t 1$, previously proven to be highly effective in the cornea (28), was amplified from the pshRNA sFLT1 plasmid along with the H1 promoter (primers TGCTCTAGATTCCCAGTCACGACGTTG and ATAATGGATCCGGAAACAGCTATGACCATG) and cloned into pCDH-CMV-MCS-EF1 $\alpha$-copGFP. The CMV promoter was removed for construction of LV-H1-sFLT1shRNA-EF1 $\alpha$-copGFP following a standard cloning protocol. Viral particles were produced using standard calcium phosphate transfection in human embryonic kidney fibroblasts (293FT), concentrated using PEG-it Virus Precipitation Solution (System Biosciences), resuspended in PBS, aliquoted, and stored at $-80^{\circ} \mathrm{C}(27,47)$. Virus titer (particles/ml) was determined using the QuickTiter Lentivirus Quantitation Kit (Cell Biolabs Inc.) as described previously $(27,47)$.

Placenta-specific expression of sFLT1 shRNA. sFlt1 expression in the placenta was knocked down using lentivirus-mediated, placenta-specific expression of sFLT1 shRNA, as described previously (27, 47). Blastocysts were transduced with either control (LV-copGFP) or sFLT1 shRNA (LV-sFLT1shRNA-copGFP). Optimal virus concentration, incubation time, selection of optimally transduced blastocysts, and the procedure for blastocyst transfer into pseudopregnant recipients $(n=18)$ have been described previously $(27,47)$. Maternal blood, urine, and tissue samples from various organs (including kidneys, livers, lungs, brains, deciduas, placentas, and fetuses) were collected on
GD12, GD15, and GD18 for RNA and protein extraction, IHC, ISH, and other histopathological analyses. Implantation rate, fetal and placental weight, and number of resorption sites were also recorded.

Endometrium-specific VEGF overexpression during pregnancy. VEGF was overexpressed in the endometrium using a new method for lentivirus-mediated gene delivery into the endometrium (see Supplemental Methods). LV-VEGF/GFP or LV-Fluc/GFP was infused into the lumens of each uterine horn after denudation of the luminal epithelium. At 5 days after virus delivery, animals $(n=18)$ were mated. Blood pressure was measured every 2 days (see Supplemental Methods), starting on GD1, and blood and urine samples were collected on GD12, GD15, and GD18. To assess kidney function, albumin (Albuwell M ELISA; Exocell) and creatinine (Exocell) levels in urine specimens were measured following the manufacturer's instructions, and the albumin/creatinine ratio in each sample was determined as described previously (48). Glomerular cellularity (determined as cell number per glomeruli) was assessed in PAS-stained sections in a blinded manner by one investigator and verified by another (pathologist) (49). In each group, 60 consecutive cortical glomeruli were evaluated. Evaluation of vascular permeability and collection of tissue samples from various organs were performed as described above. Additionally, we transferred blastocysts transduced with sFLT1 shRNA to a group of Endo-VEGF animals $(n=3)$ to examine the effects of placental sFlt1 knockdown in these animals.

RNA extraction, qPCR, ISH, IHC, Western blotting, and ELISA. These were performed using procedures, probes, primers, and antibodies described previously by us and others $(27,35,45,47)$. See Supplemental Methods for details.

Statistics. All data are expressed as mean \pm SD using at least 5 samples per experimental group $(27,45)$. The significance of differences between means was analyzed by 1-way ANOVA and 2-tailed $t$ test using SPSS software (SPSS Inc.) $(27,45)$. A P value less than 0.05 was considered significant.

Study approval. Pregnant women receiving care at the Stanford Obstetrics Clinic were enrolled in this study, after written informed consent, with an approved protocol from the Stanford University Institutional Review Board. All mouse experiments were conducted in the research animal facility at Stanford University with approved protocols from the Administrative Panel on Laboratory Animal Care of Stanford University.

\section{Acknowledgments}

We thank James Roberts (University of Pittsburgh) for his comments on the manuscript. The research was supported by NIH/NICHD grant 1R21HD068981 (to N.R. Nayak), the Children's Health Initiative at Stanford (to N.R. Nayak), the March of Dimes Birth Defects Foundation (to N.R. Nayak), the Preeclampsia Foundation (to N.R. Nayak), and Alberta Innovates-Health Solutions (to J.C. Cross).

Address correspondence to: Nihar R. Nayak, Department of Obstetrics and Gynecology, Wayne State University School of Medicine, C.S. Mott Center for Human Growth and Development, 275 E. Hancock Avenue, Detroit, Michigan 48201-1405, USA. Phone: 313.577.8910; E-mail:nnayak@med.wayne.edu.

Joyce F. Sung's present address is: Department of Obstetrics and Gynecology, University of Colorado Denver, Aurora, Colorado, USA. 
1. Ilekis JV, Reddy UM, Roberts JM. Preeclampsia - a pressing problem: an executive summary of a National Institute of Child Health and Human Development workshop. Reprod Sci. 2007;14(6):508-523.

2. Young BC, Levine RJ, Karumanchi SA. Pathogenesis of preeclampsia. Annu Rev Pathol. 2010;5:173-192.

3. Roberts JM, Rajakumar A. Preeclampsia and soluble fms-like tyrosine kinase 1. JClin Endocrinol Metab. 2009;94(7):2252-2254.

4. Zhou Y, et al. Vascular endothelial growth factor ligands and receptors that regulate human cytotrophoblast survival are dysregulated in severe preeclampsia and hemolysis, elevated liver enzymes, and low platelets syndrome. $\mathrm{Am} J$ Pathol. 2002;160(4):1405-1423.

5. Levine RJ, et al. Circulating angiogenic factors and the risk of preeclampsia. $N$ Engl J Med. 2004;350(7):672-683

6. Maynard SE, et al. Excess placental soluble fms-like tyrosine kinase 1 (sFlt1) may contribute to endothelial dysfunction, hypertension, and proteinuria in preeclampsia. JClin Invest. 2003;111(5):649-658.

7. Bergmann A, et al. Reduction of circulating soluble Flt-1 alleviates preeclampsia-like symptoms in a mouse model. JCell Mol Med. 2010;14(6):1857-1867.

8. Thadhani R, et al. Pilot study of extracorporeal removal of soluble fms-like tyrosine kinase 1 in preeclampsia. Circulation. 2011;124(8):940-950.

9. Eremina V, et al. Glomerular-specific alterations of VEGF-A expression lead to distinct congenital and acquired renal diseases. JClin Invest. 2003;111(5):707-716.

10. Jain RK. Normalization of tumor vasculature: an emerging concept in antiangiogenic therapy. Science. 2005;307(5706):58-62.

11. Saito T, et al. VEGF-A induces its negative regulator, soluble form of VEGFR-1, by modulating its alternative splicing. FEBS Lett. 2013;587(14):2179-2185.

12. Ahmad S, Ahmed A. Elevated placental soluble vascular endothelial growth factor receptor-1 inhibits angiogenesis in preeclampsia. Circ Res. 2004;95(9):884-891.

13. Nevo O, et al. Increased expression of sFlt-1 in in vivo and in vitro models of human placental hypoxia is mediated by HIF-1. Am J Physiol Regul Integr Comp Physiol. 2006;291(4):R1085-R1093.

14. Zhou CC, et al. Angiotensin II induces soluble fms-Like tyrosine kinase-1 release via calcineurin signaling pathway in pregnancy. Circ Res. 2007;100(1):88-95.

15. George EM, Cockrell K, Adair TH, Granger JP. Regulation of sFlt-1 and VEGF secretion by adenosine under hypoxic conditions in rat placental villous explants. Am J Physiol Regul Integr Comp Physiol. 2010;299(6):R1629-R1633.

16. Kupferminc MJ, et al. Vascular endothelial growth factor is increased in patients with preeclampsia. Am J Reprod Immunol. 1997; 38(4):302-306.

17. Hunter A, Aitkenhead M, Caldwell C, McCracken G, Wilson D, McClure N. Serum levels of vascular endothelial growth factor in preeclamptic and normotensive pregnancy. Hypertension. 2000;36(6):965-969.

18. Tsatsaris V, et al. Overexpression of the soluble vascular endothelial growth factor receptor in preeclamptic patients: pathophysiological consequences. J Clin Endocrinol Metab. 2003;88(11):5555-5563.

19. Baker PN, Krasnow J, Roberts JM, Yeo KT. Elevated serum levels of vascular endothelial growth factor in patients with preeclampsia. Obstet Gynecol. 1995;86(5):815-821.

20. Sharkey AM, et al. Maternal plasma levels of vascular endothelial growth factor in normotensive pregnancies and in pregnancies complicated by pre-eclampsia. Eur J Clin Invest. 1996;26(12):1182-1185.

21. Murakami Y, et al. Exogenous vascular endothelial growth factor can induce preeclampsia-like symptoms in pregnant mice. Semin Thromb Hemost. 2005;31(3):307-313.

22. Lee S, Jilani SM, Nikolova GV, Carpizo D, IruelaArispe ML. Processing of VEGF-A by matrix metalloproteinases regulates bioavailability and vascular patterning in tumors. J Cell Biol. 2005;169(4):681-691.

23. Guzman-Hernandez ML, Potter G, Egervari K, Kiss JZ, Balla T. Secretion of VEGF-165 has unique characteristics, including shedding from the plasma membrane. Mol Biol Cell. 2014;25(7):1061-1072.

24. Ozawa CR, et al. Microenvironmental VEGF concentration, not total dose, determines a threshold between normal and aberrant angiogenesis. JClin Invest. 2004;113(4):516-527.

25. Daftary GS, Taylor HS. Reproductive tract gene transfer. Fertil Steril. 2003;80(3):475-484.

26. Wang H, Xie H, Zhang H, Das SK, Dey SK. Conditional gene recombination by adenovirus-driven Cre in the mouse uterus. Genesis. 2006;44(2):51-56.

27. Fan $X$, et al. Noninvasive monitoring of placentaspecific transgene expression by bioluminescence imaging. PLoS One. 2011;6(1):e16348.

28. Ambati BK, et al. Corneal avascularity is due to soluble VEGF receptor-1. Nature. 2006;443(7114):993-997.

29. Zhao S, Gu X, Groome LJ, Wang Y. Decreased nephrin and GLEPP-1, but increased VEGF, Flt-1, and nitrotyrosine, expressions in kidney tissue sections from women with preeclampsia. Reprod Sci. 2009;16(10):970-979.

30. Miquerol L, Langille BL, Nagy A. Embryonic development is disrupted by modest increases in vascular endothelial growth factor gene expression. Development. 2000;127(18):3941-3946.

31. Cheng SY, Nagane M, Huang HS, Cavenee WK. Intracerebral tumor-associated hemorrhage caused by overexpression of the vascular endothelial growth factor isoforms VEGF121 and VEGF165 but not VEGF189. Proc Natl Acad Sci U S A. 1997;94(22):12081-12087.

32. Lorquet $\mathrm{S}$, et al. Soluble forms of VEGF receptor-1 and -2 promote vascular maturation via mural cel recruitment. FASEB J. 2010;24(10):3782-3795.

33. Hirashima M, Lu Y, Byers L, Rossant J. Trophoblast expression of fms-like tyrosine kinase 1 is not required for the establishment of the mater- nal-fetal interface in the mouse placenta. Proc Natl Acad Sci U S A. 2003;100(26):15637-15642.

34. Clark DE, et al. A vascular endothelial growth factor antagonist is produced by the human placenta and released into the maternal circulation. Biol Reprod.1998;59(6):1540-1548.

35. He Y, Smith SK, Day KA, Clark DE, Licence DR, Charnock-Jones DS. Alternative splicing of vascular endothelial growth factor (VEGF)-R1 (FLT-1) pre-mRNA is important for the regulation of VEGF activity. Mol Endocrinol.1999;13(4):537-545.

36. Rossant J, Cross JC. Placental development: lessons from mouse mutants. Nat Rev Genet. 2001;2(7):538-548.

37. Georgiades P, Ferguson-Smith AC, Burton GJ. Comparative developmental anatomy of the murine and human definitive placentae. Placenta. 2002;23(1):3-19.

38. Redline RW, Patterson P. Pre-eclampsia is associated with an excess of proliferative immature intermediate trophoblast. Hum Pathol. 1995;26(6):594-600.

39. Brosens JJ, Pijnenborg R, Brosens IA. The myometrial junctional zone spiral arteries in normal and abnormal pregnancies: a review of the literature. Am JObstet Gynecol. 2002;187(5):1416-1423.

40. Kumasawa K, et al. Pravastatin induces placental growth factor (PGF) and ameliorates preeclampsia in a mouse model. Proc Natl Acad Sci U S A. 2011;108(4):1451-1455.

41. Powers RW, et al. Maternal serum soluble fms-like tyrosine kinase 1 concentrations are not increased in early pregnancy and decrease more slowly postpartum in women who develop preeclampsia. Am JObstet Gynecol. 2005;193(1):185-191.

42. Chaiworapongsa T, et al. The maternal plasma soluble vascular endothelial growth factor receptor-1 concentration is elevated in SGA and the magnitude of the increase relates to Doppler abnormalities in the maternal and fetal circulation. JMatern Fetal Neonatal Med. 2008;21(1):25-40.

43. Pang L, et al. An increase in vascular endothelial growth factor (VEGF) and VEGF soluble receptor-1 (sFlt-1) are associated with early recurrent spontaneous abortion. PLoS One. 2013;8(9):e75759.

44. Signore C, et al. Circulating angiogenic factors and placental abruption. Obstet Gynecol. 2006;108(2):338-344.

45. Fan $X$, et al. VEGF blockade inhibits angiogenesis and reepithelialization of endometrium. FASEB J. 2008;22(10):3571-3580.

46. Conklin LD, et al. HIV-based vectors and angiogenesis following rabbit hindlimb ischemia. JSurg Res. 2005;123(1):55-66

47. Fan X, et al. Transient, inducible, placentaspecific gene expression in mice. Endocrinology. 2012;153(11):5637-5644.

48. Li Z, et al. Recombinant vascular endothelial growth factor 121 attenuates hypertension and improves kidney damage in a rat model of preeclampsia. Hypertension. 2007;50(4):686-692.

49. Al-Douahji M, Brugarolas J, Brown PA, StehmanBreen CO, Alpers CE, Shankland SJ. The cyclin kinase inhibitor $\mathrm{p} 21 \mathrm{WAF} 1 / \mathrm{CIP} 1$ is required for glomerular hypertrophy in experimental diabetic nephropathy. Kidney Int. 1999;56(5):1691-1699. 